\title{
Procedimentos de avaliação da qualidade do brincar na prática da terapia ocupacional: um estudo exploratório
}

\section{Procedures for assessing the quality of play in occupational therapy practice: an exploratory study}

\author{
Maria Inês Britto Brunello ${ }^{1}$, Fernanda Stella Risseto Mieto ${ }^{2}$, Carolina \\ Donato da Silva ${ }^{3}$
}

http://dx.doi.org/10.11606/issn.2238-6149.v24i2p95-102

\begin{abstract}
Brumello MIB, Mieto FSR, Silva CD. Procedimentos de avaliação da qualidade do brincar na prática da terapia ocupacional: um estudo exploratório. Rev. Ter. Ocup. Univ. São Paulo, 2013 maio/ago, 24(2);95-102.
\end{abstract}

RESUMO: INTRODUÇÃO: Na literatura, constata-se a escassez de estudos sobre o lúdico e o pouco uso de instrumentos de avaliação da qualidade do brincar utilizados por terapeutas ocupacionais na prática clínica. OBJETIVOS: Investigar como terapeutas ocupacionais têm realizado avaliações que procuram entender a qualidade do brincar das crianças. METODOLOGIA: Os dados foram coletados através de questionário semi-estruturado aplicados a 10 terapeutas ocupacionais que trabalhavam com crianças em diferentes serviços e propostas de intervenção na cidade de São Paulo. A análise dos dados foi realizada por estratégias qualitativas. RESULTADOS/CONCLUSÃO: Através da análise qualitativa foram criados quatro núcleos temáticos. Observou-se que nenhuma entrevistada aplica avaliação padronizada da qualidade do brincar em sua prática clínica. No entanto, todas as entrevistadas avaliam a qualidade do brincar da criança através da observação direta da atividade lúdica e entrevista não estruturada com os pais. A obtenção de dados da avaliação da qualidade do brincar é considerada significativa para todas as terapeutas ocupacionais no processo de construção de planos de intervenção.

DESCRITORES: Terapia ocupacional; Protocolos; Criança; Jogos e brinquedos.
Brumello MIB, Mieto FSR, Silva CD. Procedures for assessing the quality of play in occupational therapy practice: an exploratory study. Rev. Ter. Ocup. Univ. São Paulo, 2013 maio/ago, 24(2);95102 .

ABSTRACT: BACKGROUND: In literature, it has been confirmed not only the scarcity of studies about the activities related to play but also the instruments of evaluation of the quality of play used by occupational therapists in clinical practice. OBJECTIVES: Investigate the way occupational therapists have carried out protocols of evaluations the quality of play. METHODS: Data have been collected through semistructured questionnaire applied to ten occupational therapists who work with children in different services and intervention proposals in the city of São Paulo. Data analysis was performed by qualitative strategies. RESULTS / CONCLUSION: Through qualitative analysis were created four thematic. It was observed that no interviewed applies standardized in their clinical practice. However, all interviews assess the quality of child's play by direct observation of play activity and unstructured interview with the parents. All occupational therapists considered important to assess the quality of play to elaborate therapeutic plans.

KEYWORDS: Occupational therapy; Protocols; Child; Play and playthings.

1. Professora Doutora do Curso de Terapia Ocupacional do Departamento de Fisioterapia, Fonoaudiologia e Terapia Ocupacional da FMUSP.

2. Terapeuta Ocupacional do Curso de Terapia Ocupacional do Departamento de Fisioterapia, Fonoaudiologia e Terapia Ocupacional da FMUSP, mestre em Ciências da Saúde pela Escola de Enfermagem da USP.

3. Professora Substituta do Curso de Terapia Ocupacional da Universidade Federal de São Carlos - UFSCar e Terapeuta Ocupacional do CAPS Infantil da Vila Maria/Vila Guilherme.

Endereço para correspondência: Rua Cipotânea, 51 Cidade Universitária, São Paulo, SP. 


\section{INTRODUÇÃO}

$\mathrm{O}$ interesse pela pesquisa é decorrente da prática assistencial desenvolvida no Laboratório de Estudos sobre Deficiência e Cotidiano do Curso de Terapia Ocupacional da USP entre os anos de 1996 e 2007, no qual eram atendidas crianças com deficiência intelectual e/ou transtorno global do desenvolvimento e aliavam três vertentes: assistência, ensino e pesquisa.

O compromisso da equipe se deu no sentido de ampliar e garantir tanto a rede relacional das crianças assistidas como o engajamento destas em atividades significativas à sua faixa etária, considerando a brincadeira como fundamental atividade da infância para o desenvolvimento global e estruturação da identidade. A intervenção acontecia a partir de uma concepção de clínica da infância em que a responsabilidade profissional era redimensionar práticas que possibilitassem dar sentido à vida das crianças na trama do cotidiano, considerando seu contexto pessoal, social e cultural.

As observações realizadas durante os atendimentos demandavam dos profissionais uma sensibilidade na escuta e no olhar, implicados com todo movimento, gesto, comunicação verbal e corporal das crianças, procurando traduzir os fatos ocorridos nas entrelinhas dos acontecimentos. O brincar era compreendido como uma das principais atividades da infância a qual possibilitava à criança se apresentar ao mundo, bem como conhecê-lo. Dessa forma, era exigido do profissional um olhar sensível e, portanto afetivo, sobre a criança brincando. Preconizavase que o profissional mantivesse um olhar que pensa, reflete, interpreta e avalia.

Assim, para atingir os objetivos clínicos que respondessem às demandas de cada sujeito atendido, eram realizadas observações e avaliações criteriosas dessas crianças brincando, no sentido de compreender e reconstruir sua história lúdica, conhecendo-as na totalidade e complexidade de suas ações no mundo constituídas no cotidiano e evitandose interpretações baseadas em leituras reducionistas e desvinculadas do fazer singular de cada indivíduo.

A avaliação das crianças focava não só o desenvolvimento motor, sensorial, cognitivo, afetivo, psíquico e o seu contexto sociocultural, mas principalmente a qualidade do brincar apresentada por cada uma. Isto é, procurava-se observar nessas crianças o seu brincar espontâneo, livre, investido de sentido, pois entendíamos que, a partir dessa atividade, seria possível se aproximar de suas histórias e formas particulares de ser e estar no mundo.

A experiência desses anos proporcionou à equipe elaborar procedimentos de avaliação do brincar, de forma não padronizada, pensados a partir da convivência e interação com as crianças na clínica, contribuindo para organizar dados e registros; possibilitar planejamento de ações; reconhecer processos de evolução e mudanças; e realizar apontamentos detalhados da atividade lúdica das crianças, revelando suas potências de criação, experimentação e transformação. Neste sentido, afirma-se que um terapeuta ao ter um conjunto de dados minuciosos e bem elaborados que possibilitem compreender as informações e ações de uma criança a partir do seu brincar é capaz de propor planejamentos e intervenções que contemplem as necessidades de cada criança.

Essa prática, somada a outras experiências e estudos no campo da infância, nos instigou a pesquisar como os terapeutas ocupacionais têm realizado avaliações que procuram entender a qualidade do brincar das crianças. Estudos sobre o assunto confirmam a escassez de produção na literatura sobre o tema e o pouco uso de instrumentos de avaliação da atividade lúdica utilizados por esses profissionais na prática clínica.

Na Terapia Ocupacional, Rezende ${ }^{22}$ aponta que foi somente entre os anos de 1970 e 1980 que houve aumento de produções internacionais acerca do tema brincar e, consequente a esse fato, foram desenvolvidos instrumentos para observação de crianças brincando em ambientes clínicos. A autora apresenta protocolos de avaliação do brincar reconhecidos na literatura: Histórico Lúdico ${ }^{27}$; Escala Lúdica Pré-escolar de Knox ${ }^{16}$; Escala de Brincar da Criança (Barnett, 1990); Teste de Entretenimento ${ }^{4}$; Simbolic and Imaginative Developmental Checlist - SIP - DC (Stagnitti, 1998); The Child-Initiated Pretend Play Assessment - ChIPPA ${ }^{26} \mathrm{e}$ Avaliação do Comportamento Lúdico ${ }^{11}$.

Apesar da existência de diferentes protocolos de avaliação lúdica, Couch et al. ${ }^{9}$ e Stagnitti ${ }^{24,25}$, constatam, em pesquisas com terapeutas ocupacionais americanos e australianos, que esses profissionais raramente mencionam o uso de instrumentos de avaliação do brincar na clínica da Terapia Ocupacional.

Em nível nacional, Rezende 22 afirma que na prática dos terapeutas ocupacionais brasileiros os instrumentos de avaliação do brincar ainda são pouco utilizados e quando referidos em publicações são fundamentados em protocolos estrangeiros.

Dentre estas publicações, identificamos a pesquisa de Sant'Anna et al. (2008), que realizaram a adaptação transcultural para a população brasileira dos protocolos de avaliação do comportamento lúdico desenvolvidos pela terapeuta ocupacional canadense Ferland ${ }^{11}$. Além destas autoras, Pacciulio et al. ${ }^{19}$ publicaram estudo preliminar de adaptação transcultural da Escala Lúdica Pré-escolar da terapeuta ocupacional americana Knox ${ }^{16}$. Pfeifer et al. ${ }^{20}$ também publicaram um artigo sobre a adaptação transcultural e confiabilidade do Child-Initiated Pretend Play 
Assessment (ChIPPA) da terapeuta ocupacional australiana Stagnitti ${ }^{25}$.

Para dimensionar o uso de avaliações do brincar nas intervenções clínicas dos terapeutas ocupacionais em nível nacional realizamos levantamento de artigos nos periódicos Cadernos de Terapia Ocupacional da UFSCAR e Revista de Terapia Ocupacional da USP, entre 2007 e 2012. Esses periódicos foram selecionados por serem específicos da Terapia Ocupacional além de referência nas práticas desses profissionais. Foram utilizados os seguintes descritores: desenvolvimento infantil, avaliação, criança, pediatria, terapia ocupacional. Nesse levantamento, constatou-se a publicação de 29 artigos. Deste total de artigos analisados que trazem temas relacionados à infância, cotidiano e terapia ocupacional, apenas 13 abordam o brincar e somente um se refere ao uso de instrumento de avaliação da qualidade do brincar.

Assim, justifica-se a relevância de aprofundar estudos e pesquisas que possam contribuir com reflexões acerca dos procedimentos de avaliação da qualidade do brincar utilizados na clínica de terapeutas ocupacionais.

\section{PROCEDIMENTOS METODOLÓGICOS}

Trata-se de uma pesquisa exploratória de análise qualitativa. A coleta dos dados foi realizada a partir de entrevistas semi-estruturadas aplicadas a 10 terapeutas ocupacionais que trabalham com crianças em diferentes serviços e propostas de intervenção na cidade de São Paulo.

O critério de inclusão para participação nas entrevistas foi ser terapeuta ocupacional com pelo menos dois anos de formada em diferentes Instituições de Ensino Superior e trabalhar com crianças em serviços públicos ou privados. Consideramos que os resultados obtidos, a partir dos sujeitos entrevistados, garantiram dados para reflexão e aprofundamento do tema estudado.

As entrevistas semi-abertas foram constituídas por questões que buscavam compreender como terapeutas ocupacionais avaliam a qualidade do brincar das crianças e quais instrumentos são utilizados para atingir esse objetivo. Inicialmente, foram aplicadas duas entrevistas piloto a fim de se verificar a relevância e pertinência das indagações e a coerência com os objetivos da pesquisa.

As entrevistas foram realizadas pelos pesquisadores pessoalmente e gravadas para posterior transcrição e análise. Foram efetuados contatos prévios com os entrevistados explicando os objetivos da pesquisa. A pesquisa foi aprovada pelo Comitê de Ética da Faculdade de Medicina da Universidade de São Paulo e todos os terapeutas ocupacionais assinaram o termo de consentimento.

Para a análise dos dados, seguimos a orientação metodológica de Minayo (2004), que propõe as seguintes etapas: transcrição e leitura das informações; ordenamento dos dados para classificação em categorias e análise final.

O tratamento dos dados teve orientação de Bardin ${ }^{1}$, Martins e Bicudo ${ }^{18}$ que trabalham em uma perspectiva de análise na qual a interpretação das falas é realizada à medida que os dados vão sendo coletados. As unidades de significado das descrições foram sendo compostas e organizadas em temas de análise. O processo de análise foi realizado através de leitura exaustiva das entrevistas para identificação dos núcleos temáticos e discussão e análise dos resultados da pesquisa.

\section{RESULTADOS E ANÁLISE}

As entrevistadas são todas do sexo feminino, formadas em Terapia Ocupacional e nenhuma delas fez outra graduação. Das dez entrevistadas, duas têm um ano de experiência profissional no campo da infância, uma está nesta área há 27 anos e as outras terapeutas ocupacionais trabalham com esta população de três a sete anos.

Do total de entrevistadas, cinco profissionais não realizaram cursos de pós-graduação lato senso ou estrito senso, três têm especialização em áreas relacionadas à infância, duas fizeram aprimoramento e uma mestrado.

Os referenciais teórico-metodológicos utilizados pelas terapeutas ocupacionais entrevistadas para fundamentar sua prática e pensar a infância e o brincar na clínica apoiam-se em diferentes campos do conhecimento, como: Psicanálise, Filosofia e Psicologia. A maioria das entrevistadas utiliza a teoria Winnicottiana, seguida da Teoria do Desenvolvimento de Piaget, da Teoria Construtivista e Histórico-Social de Vygotsky e da Esquizoanálise de Deleuze e Guattari.

Em relação aos locais de trabalho, a maioria atua em instituições públicas, como Centros de Atenção Psicossocial Infantojuvenis (CAPSij), Serviços de Atenção Integral ao Dependente (SAID), Hospitais infantis, Ambulatório de Especialidades, Núcleos de Apoio à Saúde da Família (NASF) e somente duas relataram trabalhar em clínicas particulares. A população atendida incluía: deficiência intelectual, transtorno global do desenvolvimento, deficiência física, comprometimento psíquico, problemáticas relacionadas à vulnerabilidade social e o uso abusivo de substâncias psicoativas.

A partir da leitura das entrevistas, foram detectados quatro núcleos temáticos que possibilitaram compreender como é realizada a avaliação da qualidade do brincar pelos terapeutas ocupacionais, no que se refere: A) à utilização ou não de instrumentos específicos de avaliação da atividade lúdica; B) às estratégias utilizadas para a obtenção de dados sobre o brincar das crianças; C) aos aspectos observados 
sobre o brincar durante o processo de avaliação; e D) às concepções da atividade lúdica que fundamentam e orientam a observação e intervenção clínica do terapeuta ocupacional.

Com relação ao uso ou não de instrumentos de avaliação da qualidade do brincar utilizados pelos terapeutas ocupacionais (núcleo temático A) observa-se que nenhuma das entrevistadas utiliza roteiro ou protocolo específico sobre avaliação dessa atividade da criança. No entanto, foi identificado na pesquisa que os protocolos por elas utilizados na prática clínica referem-se às escalas padronizadas de avaliação do desenvolvimento global da criança, não específicas da Terapia Ocupacional ou do brincar.

Os protocolos de avaliação utilizados pelas entrevistadas foram: Escala de Comportamento Adaptativo Vineland de Sara S. Sparrow, David A. Balla e Domenic V. Ciccheti, Escala de Avaliação Global do Funcionamento (GAF) do eixo V do Manual de Diagnóstico e Estatística dos Transtornos Mentais (DSM-IV), Perfil Sensorial de Winnie Dunn, Medida de Processamento Sensorial (SPD) de Cheryl Ecker e Diane Parham e Inventário de Avaliação Pediátrica de Incapacidade (PEDI) de Stephen M. Haley, Wendy J. Coster, Larry H. Ludlow, Jane T. Haltiwanger e Peter J. Andrellos. De maneira geral os respectivos instrumentos buscam mensurar a comunicação, socialização, autonomia, desenvolvimento motor e sensorial e a participação das crianças nas atividades escolares, lúdicas e sociais (Tabelas 1 e 2).

No entanto, algumas entrevistadas referem-se utilizar roteiros não padronizados de avaliação global da criança elaborados pela própria equipe da instituição, utilizados no acolhimento a familiares e crianças e no acompanhamento do processo clínico.

Ressalta-se que em todos os protocolos acima referidos, poucos itens exploram o brincar da criança em profundidade e com a preocupação de qualificá-lo, o que coloca em questão qual seria a importância e objetivos do uso dessa atividade na prática dos terapeutas ocupacionais que lidam com a infância e quais os valores e concepções atrelados ao lúdico. Porém, segundo as entrevistadas, apesar de não trabalharem com protocolos específicos estas consideram fundamental para o planejamento da intervenção do terapeuta ocupacional a obtenção de dados sobre o brincar coletados no processo de avaliação da criança, processando informações confiáveis capazes de fundamentar as tomadas de decisão do profissional.

Esse momento de avaliação inicial da criança é referido pelas entrevistadas como: anamnese, ficha ou questionário de acolhimento, roteiro de avaliação inicial, folha de triagem ou protocolo. Todas as entrevistadas localizam este procedimento como início do contato com a criança, sua família e sua demanda. Cinco das dez entrevistadas apontaram aplicar durante todo o processo de intervenção, e não somente no momento de chegada à instituição.

Em relação às estratégias utilizadas para a obtenção de dados sobre o brincar das crianças (núcleo temático B), constatou-se que em todas as entrevistas o brincar é observado de uma forma não sistematizada em diferentes situações: três terapeutas ocupacionais observam a criança brincando sozinha sem a intervenção de um adulto; todas as terapeutas ocupacionais observam o brincar da criança na interação com o terapeuta; cinco observam o brincar da criança na interação com o cuidador; sete observam o brincar da criança na interação com outras crianças (Tabela $1)$.

\footnotetext{
"A criança pode ser avaliada individualmente ou em grupo. Se a criança irrita-se na presença de outras crianças, casos de tentativa de suicídio e sintomas depressivos, possivelmente será avaliada individualmente. Se há queixa de uma dificuldade de interação social, há possibilidade de avaliação grupal para observar como a criança interage em grupo".

"Faço a avaliação em dois momentos: a criança brincando sozinha e brincando com o terapeuta, porque vejo muita diferença do brincar da criança sozinha e do brincar com você intervindo diretamente com ela".

“...durante a avaliação você consegue perceber como é contato da criança coma a mãe. Se fica muito no colo..."

“...durante a brincadeira ficava muito de olho na interação da criança com essa família, e mais, dessa família com essa criança".
}

Em todas as entrevistas foi levantada a importância da participação dos pais ou cuidadores na obtenção de informações sobre o brincar. Os terapeutas ocupacionais buscam entender, a partir dos dados da família, quais as brincadeiras preferidas das crianças no cotidiano e com quem brincam.

Para Cavalcanti ${ }^{8}$, na avaliação do brincar, o método de observação direta e a entrevista com os pais são frequentemente preferidos por terapeutas ocupacionais, verificando-se uma tendência a entrevista não estruturada.

“...eu pergunto para mãe aonde brinca, como que brinca, que brinquedo mais gosta, como manipula o brinquedo, se brinca sozinho, se brinca com crianças da idade ou idades diferentes, se a mãe senta junto para brincar com a criança". 
Brumello MIB et al. Procedimentos de avaliação da qualidade. Rev. Ter. Ocup. Univ. São Paulo, 2013 maio/ago, 24(2);95-102.

Tabela 1 - Estratégias utilizadas para a obtenção de dados sobre o brincar das crianças

\begin{tabular}{l|c}
\hline Observação do brincar da criança sem a intervenção do adulto & 3 entrevistadas \\
\hline Observação do brincar da criança com a intervenção do terapeuta & 10 entrevistadas \\
\hline Observação do brincar da criança interagindo com o cuidador & 5 entrevistadas \\
\hline Observação do brincar da criança interagindo com outra criança & 7 entrevistadas \\
\hline Contribuição das famílias na obtenção de dados sobre o brincar & 10 entrevistas \\
\hline
\end{tabular}

Em relação ao núcleo temático $\mathrm{C}$, o que se constata é que, mesmo sem haver uma avaliação específica do lúdico, as entrevistadas apontam como fundamental para a compreensão da qualidade do brincar os seguintes aspectos: a escolha do brinquedo pela criança; a brincadeira que a criança mais gosta; relação que a criança constrói com o objeto: como interage com o brinquedo, por que escolheu esse brinquedo, como manipula o objeto; função simbólica dada ao objeto lúdico; criatividade da criança na cena lúdica; capacidade motora no manuseio dos objetos; déficits do desempenho da criança na atividade do brincar; aquisições no desenvolvimento neuropsicomotor da criança; interação lúdica que estabelece com o cuidador, terapeuta ou outra criança; exploração do espaço; tempo que permanece na atividade e como encerra a brincadeira (Tabela 2).

"Observo o que a criança traz quando ela brinca, se favorece a linguagem, a interação, quais os conteúdos que trazem, as habilidades cognitivas que estão envolvidas... observo também como é o comportamento dessa criança durante o processo de escolher o brinquedo, brincar, encerrar a brincadeira e guardar o brinquedo depois".

“...observo a questão física durante o brincar, se tem uma hemiparesia, se tem uma dificuldade motora, alguma sindrome..."

"Além de saber o que a criança gosta de fazer, eu busco saber como ela faz. Por exemplo, ela diz que adora brincar de casinha... Então, como ela brinca de casinha? Quanto tempo permanece na atividade? Como ela brinca de boneca? Faz alguma associação com a história dela? Eu procuro sempre contextualizar esta brincadeira".

"Busco observar a interação da criança com o brinquedo, a possibilidade de imaginar historias e brincadeiras e a capacidade motora no manuseio dos objetos".

Tabela 2 - Aspectos observados sobre o brincar durante o processo de avaliação

\begin{tabular}{l|c}
\hline Escolha do brinquedo pela criança & 1 entrevistada \\
\hline Brincadeira que a criança mais gosta & 4 entrevistadas \\
\hline Como manipula e interage com o brinquedo & 5 entrevistadas \\
\hline Função simbólica dada ao objeto lúdico & 4 entrevistadas \\
\hline Criatividade da criança na cena lúdica & 1 entrevistada \\
\hline Capacidade motora no manuseio dos objetos & 3 entrevistadas \\
\hline Déficits do desempenho da criança na atividade do brincar & 1 entrevistada \\
\hline Aquisições no desenvolvimento neuropsicomotor da criança & 3 entrevistadas \\
\hline Interação lúdica que estabelece com o cuidador; terapeuta ocupacional e/ou criança & 3 entrevistadas \\
\hline Exploração que a criança faz do espaço & 1 entrevistada \\
\hline Quanto tempo permanece na atividade lúdica & 1 entrevistada \\
\hline Como encerra a brincadeira & 1 entrevistada \\
\hline
\end{tabular}


Em relação ao uso da atividade lúdica na clínica da terapia ocupacional (núcleo temático D), observou-se nas respostas das entrevistadas que a maioria vincula esta atividade à função terapêutica de alcançar determinados objetivos relacionados ao desenvolvimento infantil, como: facilitar a socialização e o aprendizado; possibilitar o desenvolvimento global e a experimentação de novas situações; promover a inclusão social; treinar habilidades; garantir competências para a vida adulta; contribuir para percepção de si, sua ação no mundo e compreensão do que ocorre ao seu redor.

Gunn $^{13}$ aponta que na década de 70 há o aparecimento de trabalhos de terapeutas ocupacionais que utilizam o brincar na clínica com a finalidade de desenvolver habilidades e competências funcionais e garantir o bom desempenho na vida adulta. A partir dessa ótica, a avaliação do brincar também é considerada numa perspectiva do desenvolvimento humano, de maneira a medir as aquisições da criança em função da idade cronológica.

Por outro lado, outras entrevistadas demonstraram usar este recurso por considerarem a brincadeira uma atividade espontânea, prazerosa e vital, inerente à infância, um direito específico da criança e uma das formas fundamentais de comunicação com o mundo, contribuindo para a constituição de sua singularidade.

Os resultados acima apresentados vão de encontro com as ideias de Ferland $^{11}$ quando apresenta duas correntes de pensamento sobre o uso da brincadeira na terapia ocupacional. Na primeira, o brincar é um meio para desenvolver as funções sensoriais, motoras e cognitivas da criança. Na segunda corrente de pensamento, apresentada pela autora, o brincar não é somente um meio ou recurso terapêutico, mas também um objetivo a alcançar em terapia ocupacional. Sob esta ótica, interessa-se pela criança em sua globalidade não por uma função específica e se aborda por meio das atividades que possam ter um sentido para ela e suscitar o seu interesse no domínio do brincar.

"O brincar permite a criança adquirir habilidades sensoriais, motoras, sociais e cognitivas que garantem boa funcionalidade na adolescência e vida adulta".

\footnotetext{
“...brincar é pertencer...produzir vida, inscrever-se no mundo, numa história, é poder, é direito”.
}

"O brincar é a principal atividade da criança e se não ocorre é urgente a necessidade de ensinar e facilitar para que a criança brinque, pois, somente através desta atividade é que ocorrerá seu desenvolvimento nos mais diversos aspectos, desde motricidade a abstrações e organizações de pensamentos”.

"Através do brincar a criança se desenvolve, aprende, socializa-se....é transformador para a criança, onde a linguagem aparece, os comportamentos mudam, o brincar, para mim, é transformador por si só".

"O brincar é parte integrante e fundamental do agir da criança no mundo. Através do brincar que a criança se experimenta em uma nova situação. Compreende o que ocorre a sua volta, nos conta o que tem sentido. A percepção de si e comunicação com os outros se dá através das experiências do brincar".

O que se observa na literatura, e também na prática das terapeutas ocupacionais entrevistadas, é que a concepção sobre o brincar norteia a elaboração de protocolos de avaliação. Independente da forma como cada terapeuta ocupacional coleta suas informações sobre o desenvolvimento infantil e o brincar de cada criança, podemos concluir, a partir das falas de todas as entrevistadas, que para a obtenção dos dados é necessário sistematizar o olhar do terapeuta ocupacional para três eixos: como observar a criança brincando, o que observar quando a criança brinca e para que observar a atividade lúdica em um processo terapêutico, e que todos eles são norteados a partir das concepções sobre a atividade lúdica.

Outro dado relevante trazido por três entrevistadas é a percepção de que o lúdico ainda é uma atividade desvalorizada na sociedade e que o significado sociocultural a ela atribuído é de passatempo e de improdutividade. Os depoimentos confirmam o desconforto das terapeutas ocupacionais diante do desvalor dado ao brincar por alguns membros da equipe de saúde e da família da criança. Esses dados comprometem diretamente o fato de não se aprofundar com mais detalhes sobre as brincadeiras realizadas no cotidiano das crianças acompanhadas na clínica da terapia ocupacional.

Confirmando esta informação, Fortuna ${ }^{12}$ retoma a etimologia da palavra brincar que está relacionada à ornamento e alegria e estes conceitos em nossa cultura são associados à inconsequência, improdutividade e prazer. Segundo a autora, a vida social e produtiva coloca o brincar em um status social rebaixado, desqualificando e segregando a atividade lúdica.

"A equipe não consegue entender muito, sabe que é importante, sabe que tem que brincar, mas tem pouca apropriação da importância da brincadeira. Parece que ele é simples e aplicar um teste psicodiagnóstico é muito mais elaborado do que sentar e fazer um barquinho." 
"Eu acho que de uma forma geral o brincar é muito desvalorizado. Tanto é desvalorizado que quando a gente brinca em um serviço de saúde, o atendimento é desvalorizado. Brincar é tão, tão desvalorizado que se você brinca no atendimento, então, ele vem aqui só para brincar, vocês só brincam e vocês não fazem nada. Tamanho é o desvalor do brincar, está tão, tão desvalorizado que entra nisso, temos que resgatar tanta coisa."

"E a gente tem que provar e conversar com os pais. Precisa ficar claro o que a gente faz. Porque tem muita gente que fala: - Ah! Mas eu vou levar na Terapia Ocupacional para brincar? Vai fazer Terapia Ocupacional para brincar? Brincar se brinca em casa, na escola."

\section{CONSIDERAÇÕES FINAIS}

Após análise e reflexão, identificamos que terapeutas ocupacionais entrevistados não aplicam avaliações padronizadas da qualidade do brincar em sua prática clínica. No entanto, a atividade lúdica é abordada durante a avaliação da criança por todas as profissionais que integraram o estudo.

As entrevistadas avaliam a qualidade do brincar da

\section{REFERÊNCIAS}

1. Bardin L. Análise de conteúdo. Lisboa: Edições 70; 2008.

2. Brunello MIB. Ser lúdico: promovendo a qualidade de vida na infância com deficiência [tese]. São Paulo: Instituto de Psicologia, Universidade de São Paulo; 2001.

3. Bryse K. Narrative contribution to the play history. In: Parham LD, Fazio LS, editors. Play in occupational therapy for children. St. Louis, MO: Mosby Elsevier; 2008. p.43-54.

4. Bundy AC. Assessment of play and Leisure: Delineation of the problem. Am J Occup Ther. 1993;47(3):217-22. doi: 10.5014/ ajot.47.3.217

5. Campos LFL. Métodos e técnicas de pesquisa em psicologia. Campinas: Alínea; 2005. p.23.

6. Carvalho A, organizador. Brincar(es). Belo Horizonte: UFMG, Proesa; 2005. p.24.

7. Castro EDC, Brunello MIB, Lima EMF. Atividades humanas e terapia ocupacional. In: De Carlo MMRP, Bartalotti CC. Terapia ocupacional no Brasil. Fundamentos e perspectivas. São Paulo: Plexus; 2001. criança através da observação direta da atividade lúdica e entrevista não estruturada com os pais. Após análise das entrevistas, percebemos similaridades nas estratégias utilizadas para a obtenção de dados e nos aspectos observados sobre o brincar durante o processo de avaliação da criança.

O resultado da pesquisa nos mostra que as informações coletadas sobre o brincar durante o processo da clínica de terapeutas ocupacionais são significativas para a aplicação de planos de intervenção, independentes de como as avaliações são constituídas, e que a utilização desses instrumentos, padronizados ou não, contribuem para sistematizar os dados das entrevistas e medir maior ou menor eficácia da intervenção terapêutica ocupacional.

Conclui-se que o estudo possibilitou compreendermos como terapeutas ocupacionais avaliam a qualidade do brincar da criança e a importância da análise da atividade lúdica na prática clinica deste profissional.

Além disso, a presente pesquisa pretendeu sensibilizar para uma reflexão em relação à incorporação de instrumentos de avaliação da qualidade do brincar utilizados por terapeutas ocupacionais na sua prática clínica e para a necessidade de publicações sobre o tema, a fim de contribuírem com a ampliação e divulgação de conhecimentos no campo da infância e do lúdico.

8. Cavalcanti A. Avaliação da recreação e do lazer. In: Cavalcanti A, Galvão C. Terapia ocupacional: fundamentação e prática. Rio de Janeiro: Guanabara Koogan; 2011. p.69-73.

9. Couch KJ, Deitz JC, Kanny EM. The role of play in pediatric occupacional therapy. Am J Occup Ther. 1998;52:111-7. doi: 10.5014/ajot.52.2.111

10. Deslandes SF, Gomes R, Minayo MCS. Teoria, método e criatividade. 26a ed. Rio de Janeiro: Vozes; 2007.

11. Ferland F. O Modelo lúdico: o brincar, a criança com deficiência física e a terapia ocupacional. 3a ed. São Paulo: Roca; 2006.

12. Fortuna TR. Vida e morte do brincar. In: Ávila IS, organizador. Escola e sala de aula: mitos e ritos. Porto Alegre: Editora da UFRGS; 2004. p.47-59.

13. Gunn SL. Play as occupation: implication for the handicapped. Am J Occup Ther. 1975;29(4):222-5.

14. Haguette TMF. Metodologias qualitativas na sociologia. Petrópolis: Vozes; 1992. 
15. Kishimoto TM, organizador. O brincar e suas teorias. São Paulo: Pioneira Thomson Learning; 2002.

16. Knox S. A play scale. In: Reylly M, editor. Play as exploratory learning. Beverly Hills, CA: Sage Publications; 1974. p.24766.

17. Magalhães LC. Avaliação de terapia ocupacional: o que avaliar e como avaliar. Bol Congresso Bras Ter Ocup, Belo Horizonte; 1997.

18. Martins J, Bicudo MAV. A pesquisa qualitativa em psicologia: fundamentos e recursos básicos. São Paulo: Morães; 1994.

19. Pacciulio AM, Pfeifer LI, Santos JLF. Preliminary reliability and repeatability of the Brasilian version of the revised knox preschool play scale. Occup Ther Int. 2010;17:74-80. doi: 10.1002/oti.289.

20. Pfeifer LI, Queiroz MA, Santos JLF, Stagtnitti KE. Crosscultural adaptation and reliability of child-initiated pretend play assessment (ChiPPA). Can J Occup Ther. 2011;78:187-95. Doi 10.2182/cjot.2011.78.3.7

21. Reilly M. Play as exploratory learning. Beverly Hills: Sage Publkications; 1974.

22. Rezende MB. O brincar e a intervenção da terapia ocupacional. In: Drummond AF, Rezende, organizador. Intervenção da terapia ocupacional. Belo Horizonte: Ed. UFMG; 2008. p.25-44.

23. Smith-Case J, Miller H. Occupational therapy with children pervasive developmental disorders. Am J Occup Ther. 1999;53(5):506-13. Available from: http://ajot.aotapress.net/ content/53/5/506.full.pdf

24. Stagnitti K. Understanding play: the implications for play assessment. Austr Occup Ther J. 2004;51(3):3-12. doi: 10.1046/j.1440-1630.2003.00387.x

25. Stagnitti K, Unsworth C. The test-reliability on the childinitiated pretend play assessment. Am J Occup Ther. 2004;58(1):93-9. doi:10.5014/ajot.58.1.93

26. Stagnitti K, Unsworth C. The importance of pretend play in child development: an occupational therapy perspective. Brit J Occup Ther. 2000;63(3):121-7.

27. Takata N. Play as a prescription. In: Reilly M, editor. Play as explorating learning. Beverly Hills, CA: Sage Publications; 1974. p.209-46.

28. Takata N. The play history. Am J Occup Ther. 1969;23(4):3158.

29. Trowbly C. The issue is - anticipating the future: assessment of occupational function. Am J Occup Ther. 1993;47:253-7. doi: 10.5014/ajot.47.3.253

Recebido para publicação: 16/05/2013

Aceito para publicação: 29/07/2013 